\title{
Micromirror arrays to assess luminescent nano-objects.
}

$\operatorname{AUTHOR}(S):$

Kawakami, Yoichi; Kanai, Akinobu; Kaneta, Akio; Funato, Mitsuru; Kikuchi, Akihiko; Kishino, Katsumi

\section{CITATION:}

Kawakami, Yoichi ...[et al]. Micromirror arrays to assess luminescent nano-objects.. The Review of scientific instruments 2011, 82(5): 053905.

ISSUE DATE:

2011-05

URL:

http://hdl.handle.net/2433/160133

RIGHT:

(C) 2011 American Institute of Physics. 


\section{AIP Scientific Instruments}

\section{Micromirror arrays to assess luminescent nano-objects}

Yoichi Kawakami, Akinobu Kanai, Akio Kaneta, Mitsuru Funato, Akihiko Kikuchi et al.

Citation: Rev. Sci. Instrum. 82, 053905 (2011); doi: 10.1063/1.3589855

View online: http://dx.doi.org/10.1063/1.3589855

View Table of Contents: http://rsi.aip.org/resource/1/RSINAK/v82/i5

Published by the American Institute of Physics.

\section{Related Articles}

Efficient Cherenkov-type terahertz generation in Si-prism-LiNbO3-slab structure pumped by nanojoule-level ultrashort laser pulses

Appl. Phys. Lett. 101, 151102 (2012)

Multi-range free-electron laser with a pair of dielectric multilayer mirrors

Appl. Phys. Lett. 101, 144101 (2012)

Femtosecond pump/supercontinuum-probe setup with $20 \mathrm{kHz}$ repetition rate

Rev. Sci. Instrum. 83, 093105 (2012)

Far field subwavelength imaging of magnetic patterns

Appl. Phys. Lett. 101, 111102 (2012)

Polarization-selective color-filter Fresnel lens in polymer-stabilized cholesteric liquid crystals

J. Appl. Phys. 112, 054501 (2012)

\section{Additional information on Rev. Sci. Instrum.}

Journal Homepage: http://rsi.aip.org

Journal Information: http://rsi.aip.org/about/about_the_journal

Top downloads: http://rsi.aip.org/features/most_downloaded

Information for Authors: http://rsi.aip.org/authors

\section{ADVERTISEMENT}

\section{ORTEC MAESTRO ${ }^{\circ}$ V MCA Software}

For over two decades, MAESTRO has set the standard for Windows-based MCA Emulation.

MAESTRO Version 7.0 advances further:

New! Windows 7 64-Bit Compatibility with Connections Version 8

New! List Mode Data Acquisition for Time Correlated Spectrum Events

New! Improved Peak fit calculations

New! Improved graphics handling for multiple displays

New! Open spectrum files directly from Windows Explorer

New! Improved performance with Job Functions and display updates

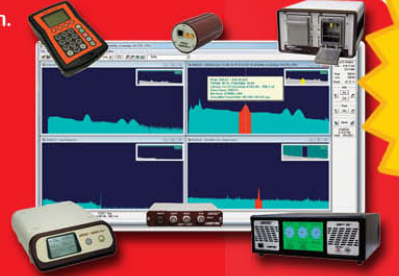

Now 64-bit

Windows 7

Compatibie! 


\title{
Micromirror arrays to assess luminescent nano-objects
}

\author{
Yoichi Kawakami, ${ }^{1,2, a)}$ Akinobu Kanai, ${ }^{1}$ Akio Kaneta, ${ }^{1}$ Mitsuru Funato, ${ }^{1,2}$ Akihiko Kikuchi, ${ }^{2,3}$ \\ and Katsumi Kishino 2,3 \\ ${ }^{1}$ Department of Electronic Science \& Engineering, Kyoto University, Kyoto 615-8510, Japan \\ ${ }^{2}$ CREST, JST, Saitama 332-0012, Japan \\ ${ }^{3}$ Department of Electrical \& Electronics Engineering, Sophia University, Tokyo 102-8554, Japan
}

(Received 23 November 2010; accepted 18 April 2011; published online 26 May 2011)

\begin{abstract}
We propose an array of submicrometer mirrors to assess luminescent nano-objects. Micromirror arrays (MMAs) are fabricated on Si (001) wafers via selectively doping Ga using the focused ion beam technique to form p-type etch stop regions, subsequent anisotropic chemical etching, and $\mathrm{Al}$ deposition. MMAs provide two benefits: reflection of luminescence from nano-objects within MMAs toward the Si (001) surface normal and nano-object labeling. The former increases the probability of optics collecting luminescence and is demonstrated by simulations based on the ray-tracing and finite-difference time-domain methods as well as by experiments. The latter enables different measurements to be repeatedly performed on a single nano-object located at a certain micromirror. For example, a single InGaN/GaN nanocolumn is assessed by scanning electron microscopy and microphotoluminescence spectroscopy. (C) 2011 American Institute of Physics. [doi:10.1063/1.3589855]
\end{abstract}

\section{INTRODUCTION}

Nanostructures such as semiconductor quantum dots, carbon nanotubes, and fullerenes have received vast interest in both science and engineering because reducing materials to the nanometer scale exploit incredible properties that cannot be realized in bulk materials. ${ }^{1}$ For example, semiconductor quantum dots fabricated by embedding tiny islands of a narrower bandgap material in a matrix with a wider energy gap can three dimensionally confine electrons and holes, and exhibit atomlike discrete energy levels. ${ }^{2,3}$ Because semiconductors involve strong optical transitions between the valence and conduction bands, their properties derived from low dimensional confinement within nanostructures have created optical devices such as single photon sources, ${ }^{4}$ lasers,${ }^{5}$ and fluorescence markers. ${ }^{6}$

One fundamental issue for nanostructures is size fluctuations because even small fluctuations significantly affect the electronic properties. Consequently, when an ensemble of nano-objects is investigated, size fluctuations often cause inhomogeneously broadened spectra, preventing the intrinsic properties of each nano-object from being assessed. Therefore, there is a strong demand for optical spectroscopy that can examine single nano-objects. Several methods, such as confocal optical microscopy ${ }^{7-9}$ and scanning near-field optical microscopy (SNOM) (Refs. 10-13), have already been demonstrated. However, performing multiple measurements on the same single nano-object remains a challenge. Hence, correlating the optical, electrical, and structural properties is difficult. We have recently developed nanospectroscopy based on SNOM and atomic force microscopy. This nanospectroscopy was used to correlate the local optical properties of InGaN quantum wells (QWs) with potential fluctuations to surface morphologies. ${ }^{13}$ This technique is capable of comprehensively assessing single nano-objects.

\footnotetext{
a)Electronic mail: kawakami@kuee.kyoto-u.ac.jp.
}

Herein, we propose a micromirror array (MMA) to assess stand-alone single nano-objects. MMAs are fabricated on $\mathrm{Si}(001)$ wafers by a maskless process based on selective Ga doping by a focused ion beam (FIB) technique and subsequent anisotropic chemical etching. The benefits brought by MMAs are twofold. First, nano-objects can be labeled and multiple measurements can be performed on the same nanoobject. Second, as demonstrated by simulations and experiments, MMAs increase the efficiency of collecting luminescence from single nano-objects. For example, a single GaN nanopillar involving an InGaN quantum disk ${ }^{14-19}$ is investigated by photoluminescence (PL) spectroscopy and scanning electron microscopy (SEM).

\section{CONCEPT AND FABRICATION OF MMA}

Figure 1 explains the MMA formation process. Si (001) wafers are initially treated with buffered hydrofluoric acid (BHF) to remove the surface oxide layers [Fig. 1(a)]. The overall process can be outlined in three basic steps: the formation of the etch stop region [Fig. 1(b)], anisotropic chemical etching [Fig. 1(c)], and $\mathrm{Al}$ deposition to form mirrorlike surfaces [Fig. 1(d)]. Thus, micromirror components with an inverse pyramidal shape are aligned to form an array with a square mesh pattern [Fig. 1(d)]. Ideally, a micromirror would hold one nano-object. Because the inverse pyramidal shape may act as a reflector of luminescence, a stronger luminescence signal is expected. Furthermore, we can identify the position of a nano-object from the mirror location. These features may facilitate multiple characterizations of single nanoobjects.

Figure 1(b) requires further explanation because the etch stop region is formed by a maskless process using FIB of Ga, unlike the conventional method based on lithography. To fully extract the MMA effects, the dimension of micromirror components should be comparable to or smaller than the focus area of the light collecting optics, as also discussed below. 


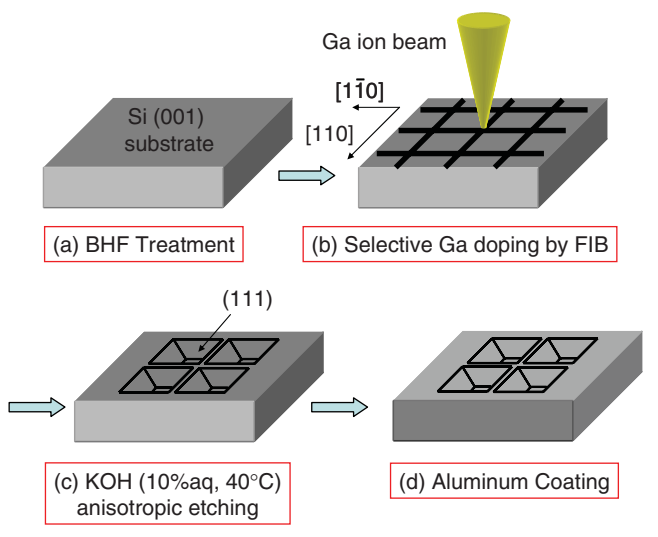

FIG. 1. (Color online) Fabrication process of MMA. (a) Removal of surface oxide by BHF, (b) selective Ga doping by FIB, (c) anisotropic Si etching by $10 \%$ aqueous $\mathrm{KOH}$ at $40^{\circ} \mathrm{C}$, and (d) $\mathrm{Al}$ deposition to create a mirror.

This is the reason why we propose to use FIB rather than photo-lithography. Although electron-beam lithography is an appropriate technique for precisely fabricating submicrometer structures, such as MMAs, it is not suitable for largescale production within a reasonable duration. On the other hand, it has been reported that $\mathrm{Ga}$ selective doping into $\mathrm{Si}$ by FIB and subsequent chemical etching can produce micro/nanostructures, where the selectively Ga doped regions act as etch stopping regions while undoped regions are etched by an alkaline solution. ${ }^{20,21}$ In this study, we adopted this technique to fabricate Si-based MMAs. Advantages of this technique over electron-beam lithography are a simpler process and minimal process duration because only line scans are necessary to form mesh-patterned Ga doped regions. The current Ga FIB conditions include an acceleration voltage of $30 \mathrm{kV}$, a current of $150 \mathrm{pA}$, and a dose of $\sim 1.5 \times 10^{15} / \mathrm{cm}^{2}$. The spot size is $\sim 40 \mathrm{~nm}$. The Monte Carlo simulations indicate that the maximum Ga concentration under these conditions is in excess of $10^{20} / \mathrm{cm}^{3}$. The FIB process typically takes $\sim 10$ min.

To effectively use the anisotropic etching characteristics of Si (001) by alkaline solutions, ${ }^{22}$ the Ga-doped etch-stop regions are aligned along the [110] direction or the (perpendicular) [1110] direction [Fig. 1(b)]. The etching rate of the $\mathrm{Si}$ $\{111\}$ plane by an alkaline solution is much slower than that of the (001) plane, ${ }^{22}$ thus as the etching proceeds, the $\{111\}$ plane predominantly appears on the etching front. Therefore, our alignment of the etch stop regions produces an inverse pyramid composed of four equivalent $\{111\}$ planes and a bottom (001) plane [Fig. 1(c)]. Herein, the alkaline solution is aqueous $\mathrm{KOH}(10 \%)$ at $40^{\circ} \mathrm{C}$. To avoid the absorption of luminescence from nano-objects by $\mathrm{Si}, \mathrm{Al}$ is deposited onto $\mathrm{Si}$ by a vacuum evaporator after the inverse pyramids are formed [Fig. 1(d)]. The Al thickness was $60 \mathrm{~nm}$ and the deposition rate was $\sim 100 \mathrm{~nm} / \mathrm{min}$.

Figure 2 displays the SEM images of the Si surfaces etched by a $\mathrm{KOH}$ solution. The development of the inverse pyramids composed of the (001) plane and four inclined planes is clearly observed. Moreover, cross sectional observations reveal that the angle between the top (001) surface
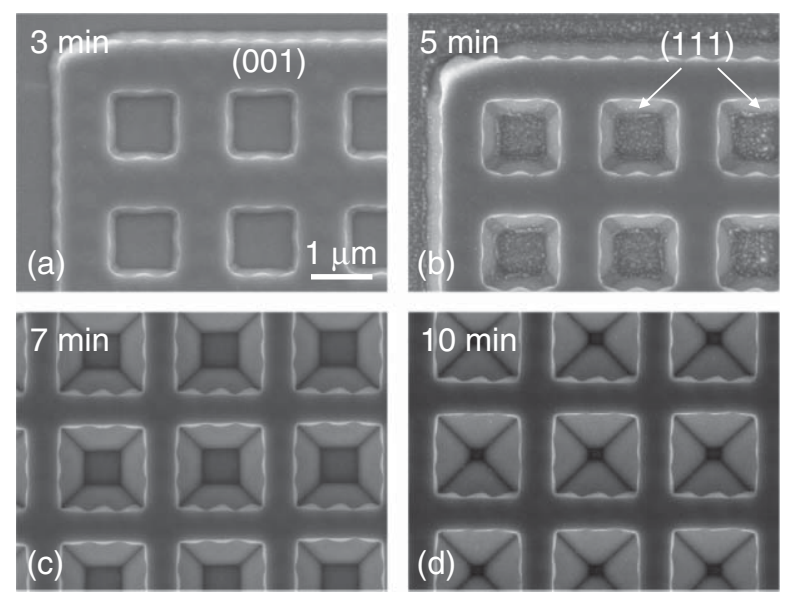

FIG. 2. SEM images of the Si surfaces etched for (a) 3, (b) 5, (c) 7, and (d) $10 \mathrm{~min}$ by $10 \%$ aqueous $\mathrm{KOH}$ at $40^{\circ} \mathrm{C}$.

and the inclined etched planes is $54.7^{\circ}$, which confirms that the inclined planes are the four equivalent $\{111\}$ planes.

\section{ENHANCEMENT OF LIGHT COLLECTION EFFICIENCY BY MMA}

The inverse pyramidal shape implies that MMAs may reflect the light created within a micromirror upward and increase the probability of the optics collecting the light. In this section, this effect is quantified by simulations and experimentally estimated. Because the micromirrors' dimensions are comparable to or may be less than the assumed luminescence wavelength of $500 \mathrm{~nm}$, we compared the ray-tracing method and the complementary finite-difference time-domain (FDTD) method.

The simulations assume that the detecting optics is an objective lens with a numerical aperture (NA) of 0.6 , which is a typical NA for microscopic PL at low temperatures using an optical window cryostat. The ratio of the light collected by the objective lens to the total emitted light at the bottom center of a micromirror is estimated. (This ratio is defined as the light collection efficiency.) Figure 3 shows the results. The horizontal axis designates the depth of a micromirror $(x)$ normalized by the distance between the surface and virtual peak of the inverse pyramid (y). Although in this particular case, the micromirror has an aperture of $2 \times 2 \mu \mathrm{m}^{2}$ on the surface, the aperture does not affect the light collection efficiency as long as the efficiency is plotted as a function of the normalized depth $(x / y)$. The ray-tracing method and the FDTD method provided almost identical results, validating the simulations. For $x=0$, that is, on a flat surface, the objective lens with an NA of 0.6 can gather only $20 \%$ of the total luminescence, whereas for a complete pyramid shape $(x / y=1)$, the coupling efficiency is nearly $90 \%$, clearly demonstrating the improved light collection efficiency due to the micromirrors.

However, it should be noted that the results in Fig. 3 are the most optimistic estimations because of the following two reasons. In the simulations, we placed a monitor plate with an aperture corresponding to NA $=0.6$ and detected light going through the monitor without considering the light propagating 


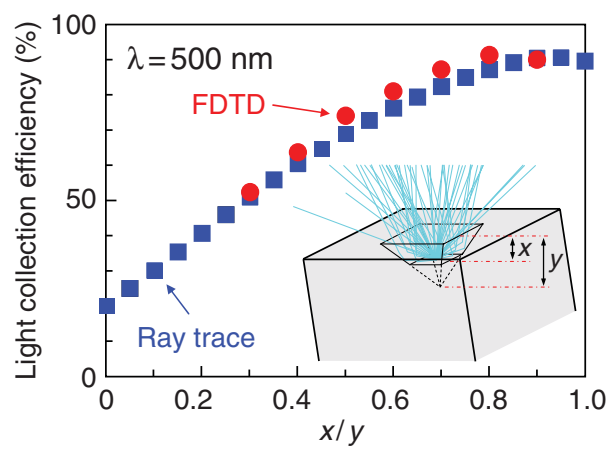

FIG. 3. (Color online) Light collection efficiency as a function of the normalized depth of a micromirror. Results from ray-tracing and FDTD methods both clearly show an increase in the collection efficiency due to micromirrors. Inset illustrates an example of a ray-tracing simulation and the definitions of $x$ and $y$.

direction, that is, without considering the light-collection cone of the objective lens. Therefore, for example, light reflected at the side wall of relatively large micromirrors may come from the outside of the cone but contribute to the calculated lightcollection efficiency. This suggests that micromirrors smaller than the objective's focus bring better performances.

Another factor that lowers the light-collection efficiency is the position of light sources. In Fig. 3, the light source was assumed to be at the bottom center of a micromirror. It was confirmed that within the bottom plane, the source position barely affects the light collection efficiency. For example, for $x / y=0.35$, that was $56 \%$ for the light source at the bottom center, while $61 \%$ at the four corners. In contrast, the light collection efficiency strongly depends on the position of the light source on the side wall, as shown in Fig. 4, where results for $x / y=0.35$ calculated by the ray-tracing method are plotted. Around the bottom plane (position 0), the efficiency is $>50 \%$ as discussed, but around the surface plane (position 1), it approaches $20 \%$, which corresponds to the light collection efficiency without MMAs. Therefore, Fig. 3 indicates the maximum light collection efficiency, while $\sim 20 \%$ is the minimum.

To confirm experimentally the enhanced light collection efficiency, luminescent latex microspheres, which are also referred to as latex beads, were dispersed on an MMA, and their luminescence properties were examined. The MMA is

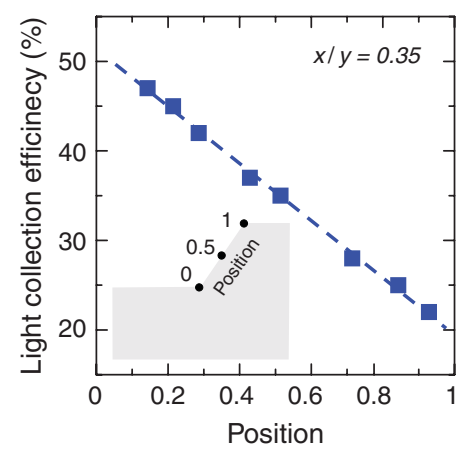

FIG. 4. (Color online) Ray-tracing calculation of light collection efficiency for a micromirror with $x / y=0.35$ as a function of the light source position on the side wall.

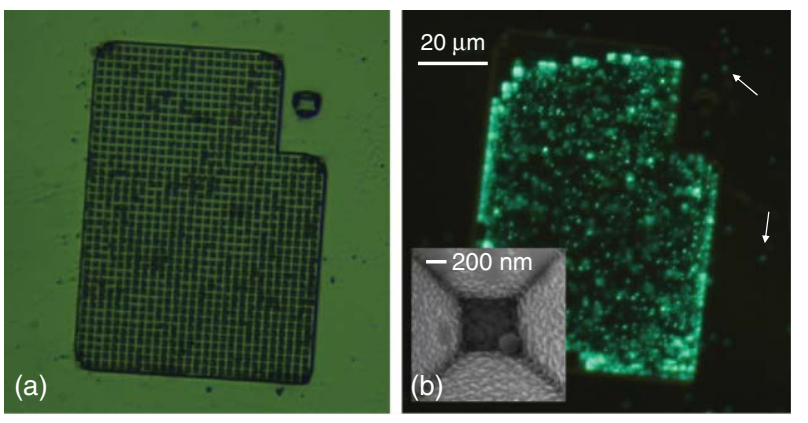

FIG. 5. (Color online) (a) Optical microscopy image of an MMA on which latex microspheres are dispersed. Inset of (b) is a SEM image showing the bottom region of a micromirror capturing a single latex microsphere. (b) Fluorescence microscopy image of the luminescence from the latex microspheres. Arrows indicate latex microspheres outside MMA.

consisted of $1.5 \times 1.5 \mu \mathrm{m}^{2}$ micromirrors. The representative diameter of the latex microspheres is $200 \mathrm{~nm}$ and the emission wavelength is $480 \mathrm{~nm}$. Figure 5(a) displays an optical microscopy image of the MMA with latex microspheres, while the inset of Fig. 5(b) shows a SEM image of a micromirror containing a single latex microsphere. The $\mathrm{Al}$ covered side walls are optically smooth, but SEM did detect roughness, suggesting further optimization of the $\mathrm{Al}$ evaporation conditions is necessary. The estimated normalized depth $(x / y)$ is 0.8 . Figure 3 predicts that the maximum light collection efficiency at this normalized depth is $80 \%-90 \%$. Figure 5(b) is a fluorescence microscopy image of the MMA where the arrows indicate latex microspheres outside of the MMA. Comparing the microspheres outside to those within the MMA confirms that MMA increases the apparent luminescence intensity as a consequence of redirecting the luminescence upward.

To quantify the increment, the PL was excited by an InGaN based laser diode emitting at $400 \mathrm{~nm}$. The PL was detected by a liquid nitrogen-cooled charge-coupled device (CCD) under a confocal setup using an objective lens with a NA of 0.6 and a single-mode fiber as a pinhole. The average PL intensity from many latex microspheres in MMAs is about 1.5 times greater than those outside MMAs. Hence, the estimated light collection efficiency is $30 \%$. The experimental increase is between the predicted maximum $(80 \%-$ $90 \%)$ and minimum $(\sim 20 \%)$. The rather low increase of $30 \%$ may be due to the dimension of the micromirror $(1.5$ $\times 1.5 \mu \mathrm{m}^{2}$ ) larger than the focus spot of the objective lens $(\sim 1 \mu \mathrm{m})$, as well as imperfect mirrors. In this study, we used the relatively large micromirrors to facilitate the fabrication process and proved the concept. In the future study, the light collection efficiency will be increased by reducing the micromirror dimension and improving the mirror quality.

\section{LABELING OF A NANO-OBJECT BY MMA - INGaN/GaN NANO-COLUMNS}

In this section, InGaN/GaN nanocolumns ${ }^{14,15,17-19}$ are used to demonstrate another benefit of MMAs, labeling of nano-objects for multiple assessments. The sample is grown on a sapphire (0001) substrate by nitrogen plasma assisted 

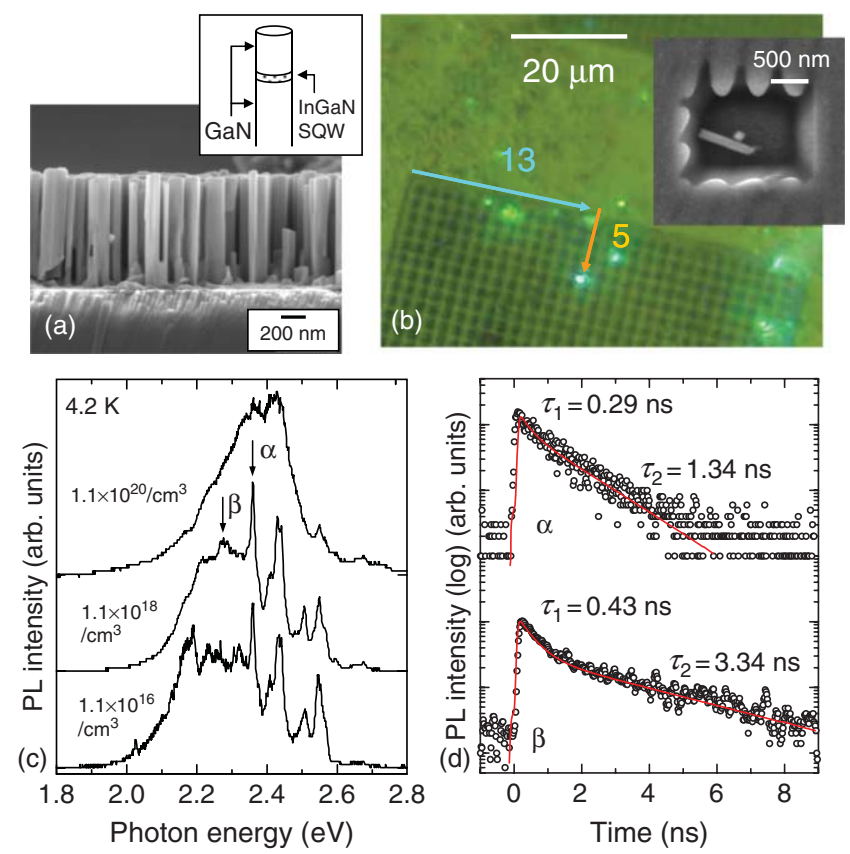

FIG. 6. (Color online) (a) Cross sectional SEM image of InGaN/GaN nanocolumns with a schematic of the structure. Inset of (b) is a SEM image of a single nanocolumn captured in a micromirror, and (b) is an optical microscopy image, on which a fluorescence microscopy image is superimposed. Single nanocolumn in the inset SEM image is located in the micromirror at an address of $(13,5)$ shown in (b). (c) PL spectra of the single nanocolumn acquired at $4.2 \mathrm{~K}$ under different excitation carrier densities. (d) PL decays monitored at emission energies of $\alpha(2.36 \mathrm{eV}, 526 \mathrm{~nm})$ and $\beta(2.27 \mathrm{eV}$, $546 \mathrm{~nm})$. Excited carrier density is $1.1 \times 10^{18} / \mathrm{cm}^{3}$.

molecular beam epitaxy, and as schematically illustrated in the inset of Fig. 6(a), consists of $1.1-\mu \mathrm{m}$ thick GaN, a 3$\mathrm{nm}$ thick InGaN single quantum well (SQW), and 55-nm thick GaN layer. Figure 6(a) is a cross sectional SEM image of the fabricated InGaN/GaN nanocolumns. SEM measurements estimate that the nanocolumns have diameters between 40 and $120 \mathrm{~nm}$. Consequently, the macroscopic PL line width is $\sim 560 \mathrm{meV}$, which is much wider than that of planar InGaN/GaN SQWs. Hence, nanospectroscopy is required to identify the characteristics of each nanocolumn.

InGaN/GaN nanocolumns are mechanically removed from the substrate and dispersed in deionized pure water. After dropping and drying this solution onto an MMA, single nanocolumns are captured into micromirrors, as demonstrated by the inset SEM image in Fig. 6(b). (The cause of the wavy side walls has yet to be clarified.) In Fig. 6(b), a fluorescence microscopy image is superimposed onto an optical microscopy image taken at the same position. It should be noted that the inset SEM image of Fig. 6(b) is obtained at a micromirror with an address of $(13,5)$ as defined in Fig. 6(b). Thus, every time this micromirror is accessed, the same nanocolumn is assessed. In fact, the inset SEM reveals that the diameter of the nanocolumn is $107 \mathrm{~nm}$, and the PL properties of this nanocolumn are investigated below.

The excitation source was a frequency-doubled, modelocked Ti:sapphire laser emitting at $400 \mathrm{~nm}$ with a pulse width of 1.5 ps. The same confocal setup as in Sec. III was used to acquire the PL spectra, but the CCD was replaced by a streak camera for time-resolved PL (TRPL). The measurements were performed at $4.2 \mathrm{~K}$, at which non-radiative recombinations can be neglected.

Figure 6(c) shows the excitation power dependence of the PL spectra of the single nanocolumn. When the initial photoexcited carrier density is less than $1.1 \times 10^{18} / \mathrm{cm}^{3}$, spiky peaks are observed, whereas higher carrier densities broaden the spectra. The spiky peaks indicate multiple emission sites within an InGaN quantum disk with a 107-nm diameter. The variation due to the excitation power suggests that localized excitons are responsible for the spiky peaks and delocalized excitons broadened the spectra; photogenerated excitons are captured by localization centers and recombine radiatively at a low excitation carrier density, but as the excitation carrier density becomes high, excitons fill the localization sites with a low density of states and escape to the delocalized states. Figure 6(c) also shows that excitation power does not influence the spiky peak positions due to the absence of piezoelectric polarization, which is consistent with our earlier report. $^{17}$

To further discuss the exciton localization and delocalization phenomena, TRPL measurements were performed for the same nanocolumn under an excited carrier density of 1.1 $\times 10^{18} / \mathrm{cm}^{3}$. It is noteworthy that MMA enables TRPL measurements of single nanocolumns, probably due to the enhanced light collection efficiency. Figure 6(d) displays the PL decays monitored at $\alpha(2.36 \mathrm{eV}, 526 \mathrm{~nm})$ and $\beta(2.27 \mathrm{eV}$, $546 \mathrm{~nm}$ ) shown in Fig. 6(c), which correspond to a spiky peak and a broad peak, respectively. The decay curves are fitted by assuming two decay components, $A_{1} \exp \left(-t / \tau_{1}\right)+A_{2} \exp (-$ $\left.t / \tau_{2}\right) .{ }^{17}$ For the spiky emission at $\alpha, \tau_{1}=0.29 \mathrm{~ns}, \tau_{2}=1.34$ ns, and $A_{1} / A_{2}=0.9$, whereas for the broad emission at $\beta$, $\tau_{1}=0.43 \mathrm{~ns}, \tau_{2}=3.34 \mathrm{~ns}$, and $A_{1} / A_{2}=2.9$. These values are comparable to those estimated by macroscopic TRPL of a nanocolumn ensemble, ${ }^{17}$ validating the current microscopic TRPL of a single nanocolumn. The estimations indicate that the broad emission $(\beta)$ is dominated by the fast decay of about a few hundreds picoseconds, whereas the spiky emission $(\alpha)$ involves a greater contribution from the slow decay of about a few nanoseconds. We confirmed that these trends are reproducible for other emissions. (Details will be published elsewhere.) It is noteworthy that due to the uncertainty principle, the lower the localization dimensionality, the longer the radiative recombination lifetime. ${ }^{23}$ Therefore, the observed trends support the assertions that the spiky peak is due to localized excitons, whereas the broad peak is due to excitons delocalized into less confined states. It is noteworthy that all the lifetimes obtained by the single nanocolumn TRPL are much faster than the typical radiative recombination lifetime of planar InGaN/GaN QWs emitting in the same green spectral region (50-100 ns). ${ }^{24}$ This confirms that strain due to the lattice mismatch between $\mathrm{InGaN}$ and $\mathrm{GaN}$ is partially relaxed at the free side wall of nanocolumns. ${ }^{16,17,25}$

In this paper, we presented the SEM and TRPL results for a single nanocolumn in a micromirror and discussed their emission properties. However, MMAs can be applied to other characterizations, such as cathodoluminescence and steadystate PL, thereby enabling an in-depth discussion of luminescent nano-objects. 


\section{SUMMARY}

MMAs are proposed to assess luminescent nano-objects. MMAs are realized by selectively doping Ga into $\mathrm{Si}$ (001) wafers using the FIB technique and subsequent anisotropic chemical etching. Simulations based on the ray-tracing method and FDTD method strongly suggest that MMAs enhance collection of luminescence from nano-objects by the optics. This effect is then experimentally confirmed. Additionally, the structural and optical assessments of a single InGaN/GaN nanocolumn demonstrate that MMAs allow nanobjects to be labeled.

${ }^{1}$ E. L. Wolf, Nanophysics and Nanotechnology (Wiley-VCH, Weinheim, 2006).

${ }^{2}$ D.Bimberg, M. Grundmann, and N. N. Ledevtsov, Quantum Dot Heterostructures (Wiley, New York, 1999).

${ }^{3}$ U. Woggon, Optical Properties of Semiconductor Quantum Dots (SpringerVerlag, Berlin, 1997).

${ }^{4}$ M. Pelton, C. Santori, J. Vuckovic, B. Y. Zhang, G. S. Solomon, J. Plant, and Y. Yamamoto, Phys. Rev. Lett. 89, 233602 (2002).

${ }^{5}$ K. Mukai, N. Ohtsuka, M. Sugawara, and S. Yamazaki, Jpn. J. Appl. Phys. 33, L1710 (1994).

${ }^{6}$ M. Bruchez, Jr., M. Moronne, P. Gin, S. Weiss, and A. P. Alivisatos, Science 286, 2312 (1999).

${ }^{7}$ S. Kimura and T. Wilson, Appl. Opt. 30, 2143 (1991).

${ }^{8}$ A. Högele, S. Seidl, M. Kroner, K. Karrai, C. Schulhauser, O. Sqalli, J. Scrimgeour, and R. Warburton, Rev. Sci. Instrum. 79, 023709 (2008).
${ }^{9}$ K. Okamoto, A. Kaneta, Y. Kawakami, S. Fujita, J. Choi, M. Terazima, and T. Mukai, J. Appl. Phys. 98, 064503 (2005).

${ }^{10}$ E. Betzig and J. K. Trautman, Science 257, 189 (1992).

${ }^{11}$ T. Saiki, K. Nishi, and M. Ohtsu, Jpn. J. Appl. Phys. 37, 1638 (1998).

${ }^{12}$ A. Kaneta, T. Mutoh, G. Marutsuki, Y. Narukawa, T. Mukai, Y. Kawakami, and Sg. Fujita, Appl. Phys. Lett. 83, 3462 (2003).

${ }^{13}$ A. Kaneta, M. Funato, and Y. Kawakami, Phys. Rev. B 78, 125317 (2008).

${ }^{14}$ M. Yoshizawa, A. Kikuchi, M. Mori, N. Fujita, and K. Kishino, Jpn. J. Appl. Phys. 36, L459 (1997).

${ }^{15}$ A. Kikuchi, M. Kawai, M. Tada, and K. Kishino, Jpn. J. Appl. Phys. 43, L1524 (2004).

${ }^{16}$ J. Ristić, C. Rivera, E. Calleja, S. Fernández-Garrido, M. Povoloskyi, and A. Di Carlo, Phys. Rev. B 72, 085330 (2005).

${ }^{17}$ Y. Kawakami, S. Suzuki, A. Kaneta, M. Funato, A. Kikuchi, and K. Kishino, Appl. Phys. Lett. 89, 163124 (2006).

${ }^{18}$ K. Kishino, T. Hoshino, S. Ishizawa, and A. Kikuchi, Electron. Lett. 44, 819 (2008).

${ }^{19}$ H. Sekiguchi, K. Kishino, and A. Kikuchi, Appl. Phys. Lett. 96, 231104 (2010).

${ }^{20}$ P. H. La Marche, R. Levi-Setti, and Y. L. Wang, J. Vac. Sci. Technol. B 1, 1056 (1983).

${ }^{21}$ I. L. Berry and A. L. Caviglia, J. Vac. Sci. Technol. B 1, 1059 (1983).

${ }^{22}$ H. Seidel, L. Csepregi, A. Heuberger, and H. Boumgörtel, J. Electrochem. Soc. 137, 3612 (1990).

${ }^{23}$ M. Sugarawa, Phys. Rev. B 51, 10743 (1995).

${ }^{24}$ K. Nishizuka, M. Funato, Y. Kawakami, Y. Narukawa, and T. Mukai, Appl. Phys. Lett. 87, 231901 (2005).

${ }^{25}$ Y. Kawakami, A. Kaneta, L. Su, Y. Zhu, K. Okamoto, M. Funato, A. Kikuchi, and K. Kishino, J. Appl. Phys. 107, 023522 (2010). 